\title{
Exogenous spatial attention shortens perceived depth
}

\author{
Wanyi Guan ${ }^{1}$ - Jiehui Qian ${ }^{1}$ \\ Published online: 21 April 2020 \\ (C) The Psychonomic Society, Inc. 2020
}

\begin{abstract}
Although spatial attention has been found to alter the subjective appearance of visual stimuli in several perceptual dimensions, no research has explored whether exogenous spatial attention can affect depth perception, which is a fundamental dimension in perception that allows us to effectively interact with the environment. Here, we used an experimental paradigm adapted from Gobell and Carrasco (Psychological Science, 16[8], 644-651, 2005) to investigate this question. A peripheral cue preceding two line stimuli was used to direct exogenous attention to either location of the two lines. The two lines were separated by a certain relative disparity, and participants were asked to judge the perceived depth of two lines while attention was manipulated. We found that a farther stereoscopic depth at the attended location was perceived to be equally distant as a nearer depth at the unattended location. No such effect was found in a control experiment that employed a postcue paradigm, suggesting that our findings could not be attributed to response bias. Therefore, our study shows that exogenous spatial attention shortens perceived depth. The apparent change in stereoscopic depth may be regulated by a mechanism involving direct neural enhancement on those tuned to disparity, or be modulated by an attentional effect on apparent contrast. This finding shows that attention can change not only visual appearance but also the perceived spatial relation between an object and an observer.
\end{abstract}

Keywords Spatial attention · Exogenous attention · Depth perception · Binocular disparity

The question of whether attention alters perception has attracted debates from early psychologists like James (1890/1950), Wundt (1874), and Helmholtz (1925) and has been investigated through the emerging studies for the past 2 decades (for reviews, see Carrasco \& Barbot, 2019). These studies often employed a spatial cue to divert either exogenous or endogenous attention to a specific location, and the perceptual results at that location were examined. Both exogenous and endogenous attention are types of spatial covert attention, which is the deployment of attention to a certain location without accompanying eye movements (Posner, 1980). Exogenous attention, which is stimulus driven and has an involuntary nature, deploys quickly and decays rapidly; conversely, endogenous attention, which deploys slowly and

Electronic supplementary material The online version of this article (https://doi.org/10.3758/s13423-020-01724-9) contains supplementary material, which is available to authorized users.

Jiehui Qian

qianjh3@mail.sysu.edu.cn

1 Department of Psychology, Sun Yat-Sen University, Guangzhou 510006, China voluntarily, decays over a longer time (Jonides, 1981; Nakayama \& Mackeben, 1989).

Evidence suggests that both types of attention can affect the visual sensitivity or behavioral performance in many visual tasks. For example, studies have shown that transient exogenous attention induced by a briefly presented spatial cue can increase contrast sensitivity (e.g., Herrmann, MontaserHouhsari, Carrasco, \& Heeger, 2010; Pestilli \& Carrasco, 2005; also see Carrasco, 2011; Maunsell, 2015, for a review) and modulate spatial resolution (e.g., Carrasco \& Barbot, 2015; Montagna, Pestilli, \& Carrasco, 2009; Yeshurun \& Carrasco, 1998; for a review, also see Anton-Erxleben \& Carrasco 2013); sustained endogenous attention can also increase contrast sensitivity (Ling \& Carrasco, 2006; Lu \& Dosher 1998) and spatial resolution (Barbot \& Carrasco, 2017; Carrasco \& Barbot, 2015; Montagna et al., 2009). In addition, both exogenous and endogenous attention can alter the appearance of many visual dimensions - for example, increasing perceived spatial frequency and gap size (Gobell \& Carrasco, 2005), enhancing apparent saturation (Fuller \& Carrasco, 2006) and contrast (Barbot \& Carrasco, 2018; Carrasco, Ling, \& Read, 2004; Itthipuripat, Chang, Bong, \& Serences, 2019; Liu, Abrams, \& Carrasco, 2009), and improving perceptual grouping (Barbot, Liu, Kimchi, \& Carrasco, 
2018). The changes in appearance with attention are suggested to be the consequence of changes in visual processing (e.g., due to attentional gain enhancement of neuronal responses in early visual cortex), mediating the effects of attention on sensitivity and performance.

Although abundant evidence has shown that attention changes visual appearance, no previous study has investigated whether depth perception can be affected by exogenous attention. The visual ability to perceive the distance of an object is known as depth perception, which is essential for us to effectively interact with the three-dimensional environment, and how we perceive the spatial relation between an object and ourselves is crucial for many higher cognitive functions. Research has shown that the visual system has distinct processing mechanisms for depth information and twodimensional (frontoparallel) distance information (Finlayson \& Golomb, 2016; Umemura, 2015), therefore, the attentional effects on visual perception and depth perception can be inherently different. Most studies on depth perception focused on various effects at a lower perceptual level of processing (e.g., Landy \& Brenner; 2001; Petrov \& Glennerster, 2004; Qian \& Petrov, 2013) and often showed that our perception for depth was fairly accurate (e.g., the stereoacuity threshold can be less than 10 arcsec; McKee \& Taylor, 2010). Past research has shown that segregation by depth could affect the allocation of attention (He \& Nakayama, 1995) and, consequently, the performance in perceptual and memory tasks (e.g., Chunharas, Rademaker, Sprague, Brady, \& Serences, 2019; Qian, Li, Wang, Liu, \& Lei, 2017; Qian, Zhang, Wang, Li, \& Lei, 2018). On the other hand, several relevant studies show that spatial attention (Xu \& Franconeri, 2012) and featurebased attention (Kawabata, 1986; Yu, Levinthal, \& Franconeri, 2017) could bias the local features of ambiguous figures (e.g., Necker cube) to be perceived as being in front. However, none has directly investigated the attentional effect on perceived depth, and therefore this important question remains unclear. In this study, we investigated whether exogenous attention could alter the perceived depth or egocentric distance (i.e., the spatial relation between an object and an observer).

The spatial cueing paradigm devised by Carrasco and colleagues (e.g., Gobell \& Carrasco, 2005) is commonly used for investigating the effect of exogenous attention on visual appearance. In this paradigm, a peripheral cue was briefly presented at the location of the subsequently appeared stimuli, and participants responded to the target feature in a secondary comparative judgment task-for example, to judge the orientation (left or right) of a Gabor patch that has a higher contrast (target feature). The visual appearance of the target feature was found to be changed at the attended stimulus's location (e.g., contrast increases). Some researchers argued that it was decision rather than perception that was biased by attention using this experimental paradigm (Schneider, 2011), and found no enhancement on contrast of the attended target using an equality judgment task (Schneider \& Komlos, 2008). However, Anton-Erxleben, Abrams, and Carrasco (2010) compared the equality paradigm and comparative paradigm and found a reliable increase in apparent contrast using both paradigms. The authors suggested that the equality judgment was more difficult (longer reaction time), noisier (larger variation in response), and prone to bias, and thus less sensitive and effective in capturing the effect of attention on perception (also see Anton-Erxleben, Abrams, \& Carrasco, 2011). Therefore, here we used a spatial precueing comparative judgment paradigm similar to Gobell and Carrasco's (2005) to investigate whether and how exogenous attention modulates depth perception in our study.

In the comparative judgement task, we asked the participants to directly respond to the perceived depth instead of using a secondary judgement task, as in Gobell and Carrasco's (2005) study, because studies found the results of direct and secondary judgements to be similar (Carrasco \& Barbot, 2019, Anton-Erxleben et al., 2010; Schneider \& Komlos, 2008). To rule out the possibility of decision bias, we adopted a postcue paradigm in addition to the precue paradigm. Depth perception here was induced by binocular disparity, which is often used in laboratories to produce stereoscopic depth.

\section{Method}

\section{Participants}

Sixteen participants (four males) between the ages of 18 and 32 years $\left(M_{\text {age }}=22.86\right.$ years $)$ took part in the study (both the precue and the postcue experiments) for payment; 14 were naïve to the purpose of the study. All participants had normal or corrected-to-normal vision. We based our sample size on the two criteria: (1) previous literature and (2) a power analysis. Previous work investigating the effect of attentional modulation on visual perception has used a range of sample size from 8 to 18 , with a typical effect size of partial $\eta^{2}=0.64$ (Barbot et al., 2018). In addition, we conducted a power analysis using G*Power (Faul, Erdfelder, Lang, \& Buchner, 2007), which revealed that at least 12 participants would be required to have a $90 \%$ power to detect an effect in our study. Written informed consent was obtained from each participant following a protocol approved by the Institutional Review Board (IRB) of Sun Yat-sen University (Guangzhou, China).

\section{Apparatus}

The stimuli were generated by Psychtoolbox using MATLAB (The MathWorks, Natick, MA), and were viewed through a Wheatstone stereoscope on a pair of 21-inch ViewSonic 
monitors with a $60 \mathrm{~Hz}$ refresh rate and $1,920 \times 1,080$ pixel resolution. Participants were seated in a dark room at a distance of $10 \mathrm{~cm}$ from the stereoscope, with their heads stabilized by a chin rest. The distance between the stereoscope and each of the monitor screens was $67 \mathrm{~cm}$; therefore, the total viewing distance was $77 \mathrm{~cm}$.

\section{Stimuli}

The stimuli sequence consisted of a fixation display, a cue display, and a line display. The fixation display contained a black fixation square $\left(0.1^{\circ} \times 0.1^{\circ}\right)$ presented centrally on a gray background $\left(20.0 \mathrm{~cd} / \mathrm{m}^{2}\right)$. The cue display contained the fixation and a black dot cue $\left(0.3^{\circ}\right.$ in diameter) presented either at fixation (neutral cue) or centered at $3.2^{\circ}$ horizontally to the left or right of the fixation (peripheral cue). Both the fixation and the cue were perceived to be on the monitor screen with no binocular disparity applied. The line display contained the fixation and two black vertical lines $(0.14 \mathrm{~cd} /$ $\mathrm{m}^{2}$ ) with a length of $1.2^{\circ}$ and a width of $0.04^{\circ}$ centered at $3^{\circ}$ horizontally to the left and right of the fixation. Note that the size of the cue was much smaller than the line stimulus, and its position did not overlap with that of the line, preventing a possible masking effect of the cue. One of the lines served as the standard, and the other served as the test (not known to participants). Throughout the experiment, there was no binocular disparity applied to the standard line. On each trial, the binocular disparity applied to the test line was randomly chosen from a set of 10 values, ranging from $-0.18^{\circ}$ to $+0.18^{\circ}$ with a step of $0.04^{\circ}$. There was always a standing disparity between the test line and the standard line (i.e., they were never equal in depth).

The peripheral cue was set to deploy attention either to the right or left of the visual field, while the neutral cue would keep one's attention at the center of the display. The cue was uninformative because the location of the cue and the locations of the standard and the test lines were randomly chosen on each trial. Here, we referred to the condition where the cue and the test line were on the same side of the visual field as test cued; the condition where the cue and the standard line were on the same side of the visual field as standard cued; and the condition where the cue was presented at the center as neutral cue.

\section{Procedure}

The procedure was adapted from Gobell and Carrasco (2005). Participants went through a screening test and two experimental sessions with 480 trials in each. In the screening test, participants viewed a fixation display for $500 \mathrm{~ms}$, followed by a line display with two lines separated by a relative disparity of $0.14^{\circ}$ for $100 \mathrm{~ms}$. They needed to judge which of the two lines appeared to be closer/farther, and were required to achieve an accuracy of above $80 \%$ within 80 trials to ensure that they could well perceive the stereoscopic depth and thus could go on with the formal experiment trials.

A diagram of the task sequence in the formal experiment was illustrated in Fig. 1. After a 500-ms presentation of fixation, a peripheral or neutral cue was presented for $70 \mathrm{~ms}$. Following a 40-ms presentation of interstimulus interval (ISI), two lines appeared for $100 \mathrm{~ms}$. The participants were asked to report which of the two lines appeared to be closer/ farther. After the response, an 800-ms blank intertrial interval was presented before the next trial. According to Nakayama and Mackeben (1989), an interval within the range from 70 to $150 \mathrm{~ms}$ between cue and stimuli onset allows one's attention to be at the peak activation during the line's presentation. Here, we used an interval of $110 \mathrm{~ms}$, consistent with Gobell and Carrasco (2005).

Participants were required to remain fixation throughout the experiment. They were told beforehand that the cue has no indication on which line was closer/farther. In order to minimize the response bias induced by the cue, we asked half of participants to report "which line appeared to be closer" and the other half to report "which line appeared to be farther" by pressing " 1 " to indicate the left line and " 3 " to indicate the right line. If response bias existed, the participants would choose the cued line more often than the uncued line in both tasks, and any possible response bias would be balanced out when we averaged the results of these two tasks. In addition, a postcue task was performed to further test the possibility of response bias. In this control experiment, the cue was presented for $70 \mathrm{~ms}$ after the presentation of stimuli and ISI. If a response bias existed, the results of postcue task and precue task were likely to be consistent. Half of participants did the precue experiment first, and the remaining participants did the postcue experiment first to balance out the ordering effect. No feedback was provided throughout the experiments.

\section{Data analysis}

Preliminary data screening showed that three participants (one did postcue experiment first and one made judgments on the farther line) had a mean accuracy of below $50 \%$ at $\pm 0.18^{\circ}$, $\pm 0.14^{\circ}$, or both in at least one of the experimental conditions. Theoretically, the accuracy at these levels should be high, as was the case for the other participants. These extreme outliers not only indicated that the three participants were careless when performing the task (because all participants achieved an accuracy of above $80 \%$ in the screening test) and would also result in significant biases when estimating the point of subjective equality (PSE), therefore the data from these three participants were excluded in the formal analysis.

The PSE, which refers to the point where the test and the standard lines were equally likely to be reported as closer, was estimated by fitting a four-parameter Weibull psychometric function (see Supplementary Materials for details). We 


\section{Procedure}

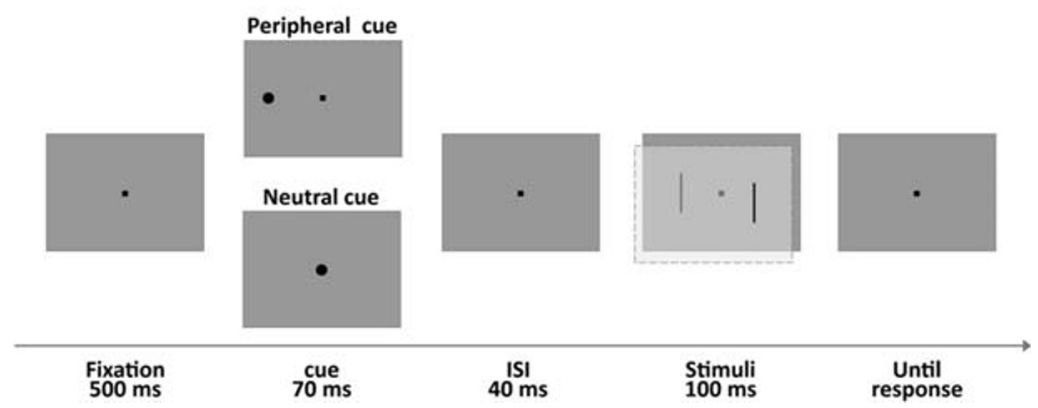

Fig. 1 Task sequence of the precue experiment. The standard line (shown in light black) had zero disparity and the test line (shown in dark black) had a disparity randomly chosen from $-0.18^{\circ}$ to $+0.18^{\circ}$. Here is an example of the standard cued condition, when the test line is closer than the standard line. The stimuli shown in the figure are not drawn to scale performed a 3 (attention condition: test cued vs. standard cued vs. neutral cue) $\times 2$ (cueing condition: precue vs. postcue) $\times 2$ (task instructions: judging closer vs. farther) mixed-design analysis of variance (ANOVA) on PSE, and GreenhouseGeisser corrections were used if the assumption of sphericity was violated. The $p$ values of post hoc tests were Bonferroni corrected.

\section{Results}

The results showed that the interaction between attention and cueing conditions was significant, $F(2,22)=12.46, p=.002$, partial $\eta^{2}=0.53$. Simple effect analysis showed that there were significant differences among PSEs of the three attention conditions in the precue experiment, $F(2,22)=11.88, p<$ .001 , partial $\eta^{2}=0.48$, whereas there was no significant difference in the postcue experiment, $F(2,22)=1.69, p=.208$, partial $\eta^{2}=0.19$. No other main effect or interaction was significant: attention condition, $F(2,22)=2.94, p=.074$, partial $\eta^{2}=0.21$; cueing condition, $F(1,11)=1.91, p=$ .195 , partial $\eta^{2}=0.15$; task instruction, $F(1,11)=0.13, p=$ .724 , partial $\eta^{2}=0.012$; task instruction $\times$ attention condition, $F(2,22)=0.58, p=.566$, partial $\eta^{2}=0.05$; task instruction $\times$ cueing condition, $F(1,11)=0.02, p=.903$, partial $\eta^{2}<0.01$; task instruction $\times$ attention condition $\times$ cueing condition, $F(2$, 22) $=0.082, p=.921$, partial $\eta^{2}<0.01$.

Because there was no significant main effect or interaction related to task instruction, the data from the two tasks were collapsed for further analyses. The psychometric functions obtained from the three attention conditions in the precue and postcue experiments are shown in Fig. $2 \mathrm{a}$ and b, respectively (see Supplementary Materials for reports on goodness of fit and the results from two participant groups that received different task instructions). In the precue experiment, the PSEs of the standard cued, neutral cue, and test cued conditions were $-0.015^{\circ},+0.006^{\circ}$ and $+0.018^{\circ}$, respectively. In other words, the test line with a disparity of $+0.018^{\circ}$ appeared to be at an equal depth as the standard line with zero disparity when attention was directed to the location of test line (test cued condition). Post hoc tests showed that there were significant differences of PSEs between the test cued and standard cued conditions, $t(12)=4.11, p=.003$, Cohen's $d=1.14$, between the test cued and neutral cue conditions, $t(12)=$ $2.49, p=.028$, Cohen's $d=0.69$, and between the standard cued and neutral cue conditions, $t(12)=2.55, p=.039$, Cohen's $d=0.71$.

In the postcue (control) experiment, although the main effect of attention was not significant, as reported above, there seems to be a response bias that the test line tended to be judged as farther than the standard line, because one-sample $t$ tests showed that the PSEs were significantly above zero under the standard cued $\left(\mathrm{PSE}=0.010^{\circ}\right), t(12)=4.74, p<$ .001 , Cohen's $d=1.31$, and neutral cue conditions (PSE = $\left.0.009^{\circ}\right), t(12)=3.60, p=.004$, Cohen's $d=1.00$. To account for the potential influence of any response biases, the PSE in the control experiment served as a baseline and was subtracted from the PSE in the precue experiment for each individual. The resulting $\triangle \mathrm{PSEs}$ were $-0.025^{\circ}$ in the standard cued condition and $+0.019^{\circ}$ in the test cued condition (shown in Fig. 3), which were significantly smaller, $t(12)=3.76, p=.003$, Cohen's $d=1.05$, and larger, $t(12)=3.56, p=.004$, Cohen's $d=0.98$, than zero, respectively. The average $\triangle$ PSE shifted about $0.022^{\circ}$ when attention was directed to either line (i.e., attention appeared to shorten the perceived depth of the test line by about $3 \mathrm{~mm}$ after any possible bias was removed). The $\triangle \mathrm{PSE}$ in neutral cue was $-0.003^{\circ}$, which was not significantly different from zero, $t(12)=0.96, p=.354$, Cohen's $d=0.27$. In addition, a repeated-measures ANOVA revealed a significant main effect of attention condition, $F(2,24)=13.70, p=$ .001 , partial $\eta^{2}=0.53$. Analysis of multiple comparisons showed significant differences of $\triangle$ PSEs between each 
(a) Pre-cue experiment
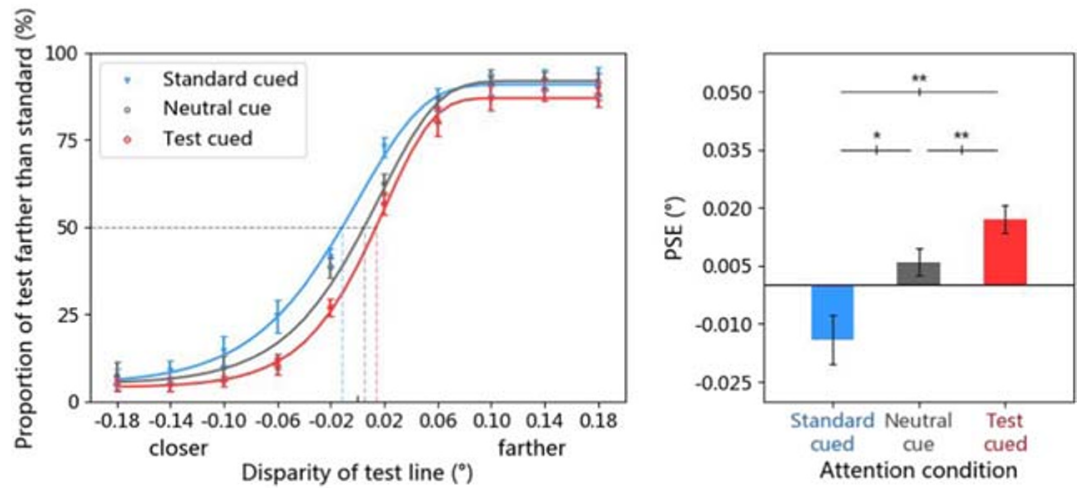

(b) Control experiment (post-cue)
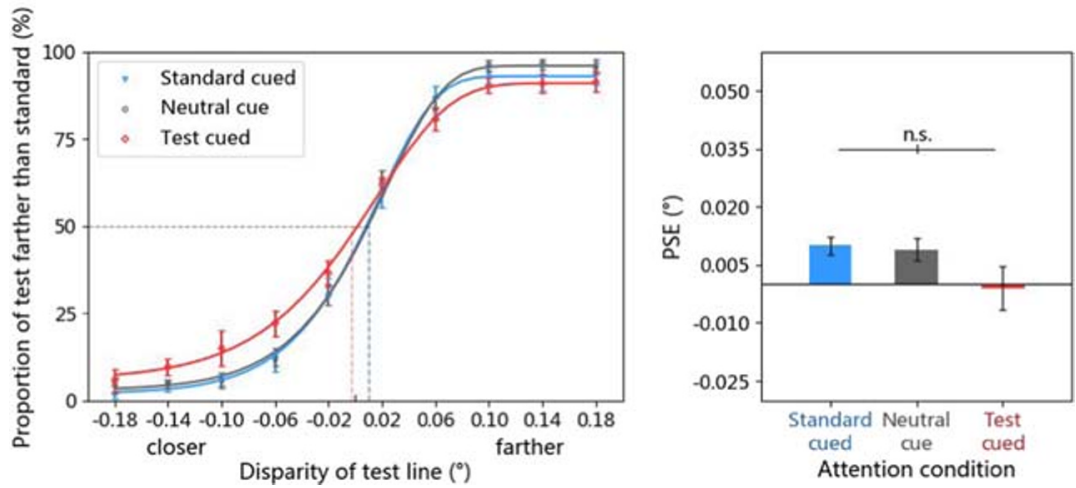

Fig. 2 Results of the precue experiment (a) and the postcue control experiment (b). Left column: Psychometric functions of the standard cued, neutral cue, and test cued conditions. Each data point is pooled across participants. Right column: Comparisons of mean PSEs among the three cue conditions. $P$-values were Bonferroni corrected. Error bars represent $\pm 1 S E M . * p<.05, * * p<.01$ comparison: for the standard cued and test cued conditions, $t(12)=4.06, p=.003$, Cohen's $d=1.13$; for the test cued and neutral cue conditions, $t(12)=4.43, p=.003$, Cohen's $d=$ 1.23; and for the standard cued and neutral cue conditions, $t(12)=2.61, p=.023$, Cohen's $d=0.73$.

\section{Discussion}

In the current study, we investigated whether and how exogenous spatial attention alters perceived depth. The results of the precue experiment showed a small but reliable shift in the perceived depth of target when it was being attended: cueing a test line with an uncrossed disparity (farther) caused it to be perceived at an equal depth as the standard line with zero disparity, and cueing the standard line with zero disparity caused it to be perceived at an equal depth as the test line with a crossed disparity (closer). The results of the postcue experiment suggested that this effect was not due to response biases induced by a cue. We further analyzed the $\triangle$ PSE between the precue and postcue experiment to assess the effect of attention on perceived depth free of the potential influence of response biases. The results confirmed that attention shortened perceived depth.

Our study is the first to demonstrate that attention affects perceived depth. This simple but important phenomenon, that attention alters the perceived spatial relation between an object and an observer, can fundamentally influence many perceptual and cognitive functions. Because our study directly assessed the effects of attention on perceived depth, we suggest that our findings can account for the previously reported attentional effect on perceived ambiguity of depth that attended local features appear to be "in front" (Kawabata, 1986; Xu \& Franconeri, 2012; Yu et al., 2017). In addition, we propose that the attentional effect on depth perception arises from three possible mechanisms. First, changes in apparent contrast may modulate perceived depth. Apparent contrast serves as a depth cue called aerial perspective (O'Shea, Blackburn, \& Ono, 1994), and previous research shown that reduced contrast is associated with judging an object as more distant (Rohaly \& Wilson, 1999). On the other hand, studies have shown that attention increases apparent contrast (Carrasco et al., 2004) by boosting early sensory processing in the visual cortex, particularly in the fusiform gyrus 


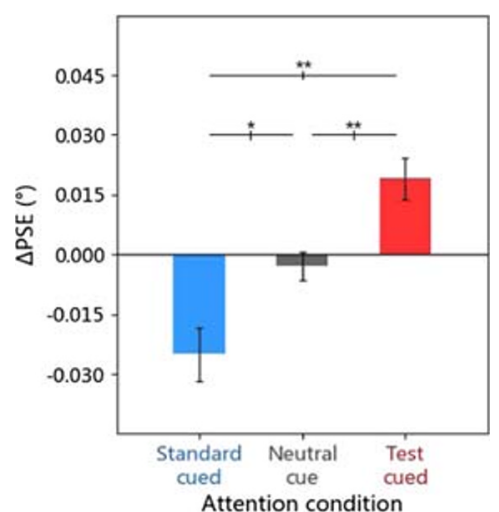

Fig. 3 Comparisons of relative shifts $(\triangle \mathrm{PSE}=$ Precue PSE - Postcue PSE) among the three cue conditions. $P$-values were Bonferroni corrected. Error bars represent \pm 1 SEM. $* p<.05, * * p<.01$

extending from the ventral surface of the occipital lobe anteriorly to the temporal lobe (Störmer, McDonald, \& Hillyard, 2009). In our study, as apparent contrast of the attended line increases, its perceived depth can be consequently decreased, and vice versa for the unattended line.

Second, the apparent change in stereoscopic depth may share a common mechanism with contrast sensitivity through the attentional effect on spatial frequency. Researchers proposed that there are at least six spatial frequency channels for detecting luminance contrast (Wilson \& Gelb, 1984), and among which three are shared with, and possibly mainly used for, stereoscopic depth processing (Shioiri, Hatori, Yaguchi, \& Kubo, 1994). In other words, although luminance contrast and stereoscopic depth are often considered to be processed independently and the channels for luminance are not identical to those for stereopsis, the two processes may have common spatial frequency channels. Shioiri et al. (1994) suggested that there are cortical cells that differ in spatial frequency tuning at an early stage of visual processing (spatial frequency channels), whereas the division of processing for luminance and stereoscopic depth seems to occur at a later stage. Therefore, as perceived spatial frequency changes with attention (Gobell \& Carrasco, 2005), sensitivity to contrast detection varies (Blakemore \& Campbell, 1969), and so does sensitivity to stereoscopic depth. The improved sensitivity to stereoscopic depth may enhance the neural response pattern towards a crossed direction and consequently make the attended stimuli to appear closer.

Furthermore, attention may directly affect the response pattern of neurons that are tuned to disparity. Signal enhancement hypothesis states that perceptual sensitivity can be enhanced by selectively attending to certain spatial location (Bashinski \& Bacharach, 1980). Indeed, evidence from electrophysiological and functional imaging studies shows that neural activities in the visual cortex increase with attention (Brefczynski \& DeYoe, 1999; Gandhi, Heeger, \& Boynton, 1999). Consistent with this hypothesis, behavioral findings showing that attention can affect spatial resolution (Carrasco
\& Barbot, 2015; Yeshurun \& Carrasco, 1998) as well as perceived spatial dimensions (Abrams et al., 2010; AntonErxleben, Henrich, \& Treue, 2007; Gobell \& Carrasco, 2005; Kirsch et al., 2018; also see the review by Carrasco \& Barbot, 2019) have been linked to preferential enhancement of neurons tuned to higher spatial frequency at the attended location (Barbot, 2016; Barbot \& Carrasco, 2017; Carrasco, Loula \& Ho, 2006; also, see the review by Anton-Erxleben \& Carrasco, 2013). Similar to the attentional effect on spatial frequency, because physiological evidence found that there are neurons in V4 that respond both for binocular disparity and spatial frequency, and their tuning are correlated (Kumano, Tanabe, \& Fujita, 2008), attention may directly change the pattern of neuron activity by shifting the disparity-tuning curve towards a crossed direction. In other words, an attended stimulus with zero-disparity or even uncrossed-disparity may evoke neural response similar to that which would be observed for a stimulus with crosseddisparity in the absence of attention.

Past research has shown that attention can alter the perceived size of an object (Anton-Erxleben et al., 2007; Gobell \& Carrasco, 2005; Kirsch, Pfister, \& Kunde, 2020; Tsal \& Shalev, 1996), and one might suspect that the attentional effect on perceived size could account for the shortened perceived depth because relative size is putatively a powerful depth cue. However, we think that this not the case for the following reasons. First, Gobell and Carrasco (2005) found that attention increases the perceived gap size of Landolt-squares, where the gap is determined by an opening between two line elements. In order for the gap size to appear bigger, the perceived size of line elements should decrease. In other words, attention increases the perceived gap size and meanwhile decreases the perceived size of the line. This phenomenon is also supported by Tsal and Shalev (1996). However, relative size suggests that an object with a smaller size is perceived to be farther (Sousa, Brenner, \& Smeets, 2011), and therefore the effect of attention on size perception predicts an opposite direction as the attentional effect on perceived depth observed in our study. Second, Tsal and Shalev (1996) suggested that attention increases the precision of size perception by narrowing attentional receptive fields (ARFs). They proposed that the metric for unattended stimuli is composed of larger ARFs and that the final output is mediated by rounding up processes, so that the unattended line is perceived as being longer than the attended one. The explanation of increased spatial resolution by attention is supported by both behavioral and neural evidence in various spatial tasks (see Anton-Erxleben \& Carrasco, 2013, for review; Carrasco \& Barbot, 2015). However, the shortened perceived depth found in our study cannot be explained by the account of increased spatial resolution because varying spatial resolution does not change the 2-D locations of the lines in the left-eye and right-eye images (i.e., the disparity of the line would remain regardless of 
attention). Therefore, the increase in spatial resolution and consequently the decrease in length perception when being attended to cannot be, if not counteracted with, the mechanism for shortening the perceived depth.

Although there was no significant difference among PSEs in the postcue experiment, the positive mean PSEs in the standard cued and neutral cue conditions indicated a possible response bias that the test line tended to be judged as farther than the standard line. It is unclear why such a bias has occurred. Because research shows that the perceived depth tends to be overestimated at near distances (Foley, 1980), one may expect that both the test line and standard line would be overestimated with the current experimental setting. Because the stereoscopic depth of the test line varied across trials, participants may experience greater uncertainty for judging its perceived depth, compared with the standard line that was fixed at zero disparity. We speculate that the greater uncertainty aggravates the overestimation, especially under the unattended (standard cued \& neutral cue) conditions. Under the attended (test cued) condition, the bias was negligible. It is possible that attending to the test line reduced its perceived uncertainty, and therefore the judgment was more accurate. Removing the response bias by calculating the difference in PSE between the precue and postcue experiments revealed almost symmetrical shifts in PSE under the test cued condition and standard cued condition. This indicates that the attentional effect on perceived depth is equivalent no matter which line was cued.

We often say, "Pay close attention." In this study, we provide solid evidence that an attended object indeed appears closer. The apparent change in depth may be modulated by an attentional effect on apparent contrast, or be regulated by a mechanism similar to that of the attentional effect on spatial frequency, such as direct neural enhancement on those tuned to disparity. This finding shows that exogenous spatial attention can change not only visual appearance but also the perceived depth or distance between a target and an observer.

Acknowledgements This work has been supported by the National Natural Science Foundation of China (31500919). The authors have no competing financial interests that might be perceived to influence the results and/or discussion reported in this paper.

Open practices statement The data and materials for all experiments are available upon request, and none of the experiments was preregistered.

\section{References}

Anton-Erxleben, K., Abrams, J., \& Carrasco, M. (2010). Evaluating comparative and equality judgments in contrast perception: Attention alters appearance. Journal of Vision, 10(11), 6-6.
Anton-Erxleben, K., Abrams, J., \& Carrasco, M. (2011). Equality judgments cannot distinguish between attention effects on appearance and criterion: A reply to Schneider (2011). Journal of Vision, 11(): $8,1-8$.

Anton-Erxleben, K., \& Carrasco, M. (2013). Attentional enhancement of spatial resolution: Linking behavioural and neurophysiological evidence. Nature Reviews Neuroscience, 14, 188-200.

Anton-Erxleben, K., Henrich, C., \& Treue, S. (2007). Attention changes perceived size of moving visual patterns. Journal of Vision, 7(11), 5.

Barbot, A. (2016). How attention enhances spatial resolution: Preferential gain enhancement of high spatial frequency neurons. Journal of Neuroscience, 36, 12080-12082.

Barbot, A., \& Carrasco, M. (2017). Attention modifies spatial resolution according to task demands. Psychological Science, 28(3), 285-296.

Barbot, A., \& Carrasco, M. (2018). Emotion and anxiety potentiate the way attention alters visual appearance. Scientific Reports, 8(1), 1-10.

Barbot, A., Liu, S., Kimchi, R., \& Carrasco, M. (2018). Attention enhances apparent perceptual organization. Psychonomic Bulletin \& Review, 25(5), 1824-1832.

Bashinski, H. S., \& Bacharach, V. R. (1980). Enhancement of perceptual sensitivity as the result of selectively attending to spatial locations. Perception \& Psychophysics, 28(3), 241-248.

Blakemore, C., \& Campbell, F. W. (1969). On the existence of neurones in the human visual system selectively sensitive to the orientation and size of retinal images. The Journal of Physiology, 203(1), 237260. doi:https://doi.org/10.1113/jphysiol.1969.sp008862

Brefczynski, J. A., \& DeYoe, E. A. (1999). A physiological correlate of the 'spotlight' of visual attention. Nature Neuroscience, 2(4), 370.

Carrasco, M. (2011). Visual attention: the past 25 years. Vision Research, 51(13), 1484-1525.

Carrasco, M., \& Barbot, A. (2015). How attention affects spatial resolution. Cold Spring Harbor Symposia on Quantitative Biology, 79, 149-160. doi:https://doi.org/10.1101/sqb.2014.79.024687

Carrasco, M., \& Barbot, A. (2019). Spatial attention alters visual appearance. Current Opinion in Psychology, 29, 56-64.

Carrasco, M., Ling, S., \& Read, S. (2004). Attention alters appearance. Nature Neuroscience, 7, 308-313.

Chunharas, C., Rademaker, R. L., Sprague, T. C., Brady, T. F., \& Serences, J. T. (2019). Separating memoranda in depth increases visual working memory performance. Journal of Vision, 19(4), 1-16.

Faul, F., Erdfelder, E., Lang, A.-G., \& Buchner, A. (2007). G*Power 3: A flexible statistical power analysis program for the social, behavioral, and biomedical sciences. Behavior Research Methods, 39, 175-191.

Finlayson, N. J., \& Golomb, J. D. (2016). Feature-location binding in 3D: Feature judgments are biased by 2D location but not position-indepth. Vision Research, 127, 49-56.

Foley, J. M. (1980). Binocular distance perception. Psychological Review, $87(5), 411$.

Fuller, S., \& Carrasco, M. (2006). Exogenous attention and color perception: Performance and appearance of saturation and hue. Vision Research, 46(23), 4032-4047.

Gandhi, S. P., Heeger, D. J., \& Boynton, G. M. (1999). Spatial attention affects brain activity in human primary visual cortex. Proceedings of the National Academy of Sciences, 96(6), 3314-3319.

Gobell, J., \& Carrasco, M. (2005). Attention alters the appearance of spatial frequency and gap size. Psychological Science, 16(8), 644651.

He, Z. J., \& Nakayama, K. (1995). Visual attention to surfaces in threedimensional space. Proceedings of the National Academy of Sciences, 92, 11155-11159.

Helmholtz, H. V. (1925). Treatise on physiological optics. Rochester, NY: Optical Society of America.

Herrmann, K., Montaser-Kouhsari, L., Carrasco, M., \& Heeger, D. J. (2010). When size matters: Attention affects performance by contrast or response gain. Nature Neuroscience, 13(12), 1554. 
Itthipuripat, S., Chang, K. Y., Bong, A., \& Serences, J. T. (2019). Stimulus visibility and uncertainty mediate the influence of attention on response bias and visual contrast appearance. Journal of Vision, 19(14), 8-8.

James, W. (1950). The principles of psychology. New York: Holt. (Original work published 1890)

Jonides, J. (1981). Voluntary versus automatic control over the mind's eye's movement. In J. Long \& A. Baddeley (Eds.), Attention and performance IX (pp. 187-203). Hillsdale, NJ: Erlbaum.

Kawabata, N. (1986). Attention and depth perception. Perception, 15(5), $563-572$.

Kirsch, W., Pfister, R., \& Kunde, W. (2020). On why objects appear smaller in the visual periphery. Psychological Science, 31(1), 88-96.

Kumano, H., Tanabe, S., \& Fujita, I. (2008). Spatial frequency integration for binocular correspondence in macaque area V4. Journal of Neurophysiology, 99(1), 402-408.

Landy, M. S., \& Brenner, E. (2001). Motion-disparity interaction and the scaling of stereoscopic disparity. In L. R. Harris \& M. Jenkin (Eds.), Vision and attention (pp. 129-150). New York, NY: SpringerVerlag.

Ling, S., \& Carrasco, M. (2006). Sustained and transient covert attention enhance the signal via different contrast response functions. Vision Research, 46(8/9), 1210-1220.

Liu, T., Abrams, J., \& Carrasco, M. (2009). Voluntary attention enhances contrast appearance. Psychological Science, 20(3), 354-362.

Lu, Z. L., \& Dosher, B. A. (1998). External noise distinguishes attention mechanisms. Vision Research, 38, 1183-1198.

Maunsell, J. H. (2015). Neuronal mechanisms of visual attention. Annual Review of Vision Science, 24(1), 373-391. doi:https://doi.org/10. 1146/annurev-vision-082114-035431

McKee, S. P., \& Taylor, D. G. (2010). The precision of binocular and monocular depth judgments in natural settings. Journal of Vision, 10(10):5, 1-13.

Montagna, B., Pestilli, F., \& Carrasco, M. (2009). Attention trades off spatial acuity. Vision Research, 49(7), 735-745.

Nakayama, K., \& Mackeben, M. (1989). Sustained and transient components of focal visual attention. Vision Research, 29(11), 1631-1647.

O'Shea, R. P., Blackburn, S. G., \& Ono, H. (1994). Contrast as a depth cue. Vision Research, 34(12), 1595-1604.

Pestilli, F., \& Carrasco, M. (2005). Attention enhances contrast sensitivity at cued and impairs it at uncued locations. Vision research, 45(14), $1867-1875$.

Petrov, Y., \& Glennerster, A. (2004). The role of a local reference in stereoscopic detection of depth relief. Vision Research, 44(4), 367 76.

Posner, M. I. (1980). Orienting of attention. Quarterly Journal of Experimental Psychology, 32(1), 3-25.

Qian, J., Li, J., Wang, K., Liu, S., \& Lei, Q. (2017). Evidence for the effect of depth on visual working memory. Scientific Reports, 7(1), 6408. doi:https://doi.org/10.1038/s41598-017-06719-6
Qian, J., \& Petrov, Y. (2013). Depth perception in the framework of general object constancy. Journal of Vision, 13, 7. doi:https://doi. org $/ 10.1167 / 13.11 .7$

Qian, J., Zhang, K., Wang, K., Li, J., \& Lei, Q. (2018). Saturation and brightness modulate the effect of depth on visual working memory. Journal of Vision, 18(9):1-12.

Rohaly, A. M., \& Wilson, H. R. (1999). The effects of contrast on perceived depth and depth discrimination. Vision Research, 39(1), 9 18 .

Schneider, K. A. (2011). Attention alters decision criteria but not appearance: A reanalysis of Anton-Erxleben, Abrams, and Carrasco (2010). Journal of Vision, 11(13):7, 1-8.

Schneider, K. A., \& Komlos, M. (2008). Attention biases decisions but does not alter appearance. Journal of vision, 8(15), 3-3.

Shioiri, S., Hatori, T., Yaguchi, H., \& Kubo, S. (1994). Spatial frequency channels for stereoscopic depth perception. Optical Review, 1(2), 311-313.

Sousa, R., Brenner, E., \& Smeets, J. B. (2011). Judging an unfamiliar object's distance from its retinal image size. Journal of Vision, 11(9), $10-10$.

Störmer, V. S., Mcdonald, J. J., \& Hillyard, S. A. (2009). Cross-modal cueing of attention alters appearance and early cortical processing of visual stimuli. Proceedings of the National Academy of Sciences of the United States of America, 106(52), 22456-22461.

Tsal, Y., \& Shalev, L. (1996). Inattention magnifies perceived length: The attentional receptive field hypothesis. Journal of Experimental Psychology: Human Perception and Performance, 22(1), 233243. doi:https://doi.org/10.1037//0096-1523.22.1.233

Umemura, H. (2015). Independent effects of 2-D and 3-D locations of stimuli in a 3-D display on response speed in a Simon task. Frontiers in Psychology, 6, 1302. doi:https://doi.org/10.3389/fpsyg.2015. 01302

Wilson, H. R., \& Gelb, D. J. (1984). Modified line-element theory for spatial-frequency and width discrimination. Journal of the Optical Society of America A, 1(1), 124-131. doi:https://doi.org/10.1364/ JOSAA.1.000124

Wundt, W. M. (1874). Grundzüge der physiologischen Psychologie (Vol. 1) [Basics of physiological psychology, Vol. 1]. Leipzig, Germany: W. Engelman.

$\mathrm{Xu}$, Y., \& Franconeri, S. L. (2012). The head of the table: Marking the "front" of an object is tightly linked with selection. Journal of Neuroscience, 32(4), 1408-1412

Yeshurun, Y., \& Carrasco, M. (1998). Attention improves or impairs visual performance by enhancing spatial resolution. Nature, 396(6706), 72-75. doi:https://doi.org/10.1038/23936

Yu, D., Levinthal, B., \& Franconeri, S. L. (2017). Feature-based attention resolves depth ambiguity. Psychonomic Bulletin \& Review, 24(3), 804-809.

Publisher's note Springer Nature remains neutral with regard to jurisdictional claims in published maps and institutional affiliations. 